Supporting Information

\title{
Controllable Thermal Oxidation and Photoluminescence Enhancement in Quasi-1D van der Waals $\mathrm{ZrS}_{3}$ Flakes
}

Junqing Guo ${ }^{\ddagger}$ a, b, Jun Tao a, b, Zhouyang Zhang a, b, Linfeng Fei a, b, Ding Li c, Jakub Jadwiszczak ${ }^{\mathrm{d}}$, Xiting Wang e , Yuzheng Guo ${ }^{\mathrm{e}}$, Xiaxia Liao ${ }^{\mathrm{a}, \mathrm{b}}$, Yangbo Zhou* ${ }^{*}$, b

*E-mail: yangbozhou@ncu.edu.cn

a School of Materials Science and Engineering, Nanchang University, Nanchang, Jiangxi, 330031, People's Republic of China

b Jiangxi Engineering Laboratory for Advanced Functional Thin Films and Jiangxi Key Laboratory for Two-Dimensional Materials, Nanchang University, Nanchang, Jiangxi, 330031, People's Republic of China

${ }^{c}$ Beijing Institute of Nanoenergy and Nanosystems, Chinese Academy of Sciences, Beijing, 100083, People's Republic of China

d Department of Electrical Engineering, Columbia University, 500 W 120th St., New York, NY, 10027, United States

e School of Electrical Engineering and Automation, Wuhan University, Wuhan, Hubei, 430072, People's Republic of China

\section{Evaluating the local heating effect during Raman spectra collection}

To evaluate the locating heating effect during Raman spectra collection, we collected the Raman spectra of $\mathrm{ZrS}_{3}$ under different laser excitation powers, with the sample placed in vacuum. Figure S1(a) shows the Raman spectra collected from a $\sim 30 \mathrm{~nm}$ thick $\mathrm{ZrS}_{3}$ flake under different laser excitation powers from $0.1 \mathrm{~mW}$ to $5 \mathrm{~mW}$ (1800 lpmm grating, $40 \mathrm{~s}$ collection time). The characteristic $A_{g}$ modes in these spectra are quite similar. No significant peak shifts are observed, as presented in Figure S1(b). Meanwhile, the corresponding optical image shows the appearance of dark spots (indicated by white arrows in Figure S3(c)) after spectra collection. These spots can be observed under laser power of $3 \mathrm{~mW}$ and $5 \mathrm{~mW}$, indicating the material degradation due to high laser power. Since we are always using a low laser power below 0.3 $\mathrm{mW}$, the local heating effects can be ignored in our reported experiments. 

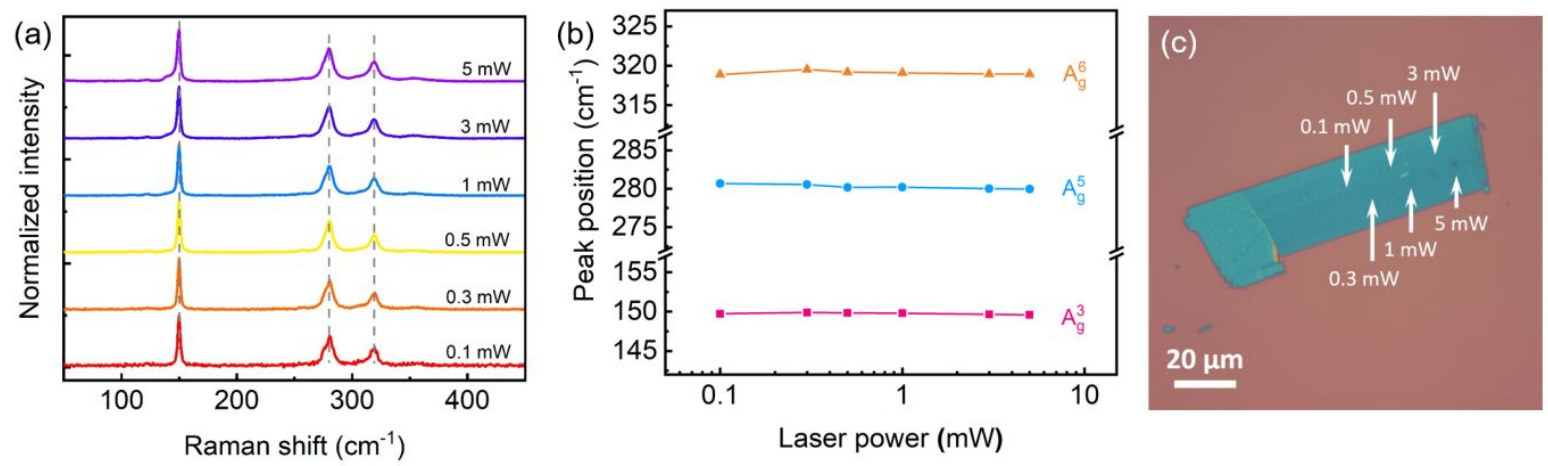

Figure S1. (a) Raman spectra of $\mathrm{ZrS}_{3}$ collected under different laser excitation powers of $0.1 \mathrm{~mW}, 0.3$ $\mathrm{mW}, 0.5 \mathrm{~mW}, 1 \mathrm{~mW}, 3 \mathrm{~mW}$ and $5 \mathrm{~mW}$, respectively. The spectra are normalised for comparison. (b) Peak positions of characteristic $A_{g}$ modes under different laser powers. No significant peak shifts are observed. (c) The corresponding optical image of the $\mathrm{ZrS}_{3}$ flake used for laser power-dependent Raman spectra collection. The white arrows indicate the dwell positions of the laser, which are insignificant at low laser powers, and can be observed at high powers of 3-5 $\mathrm{mW}$.

\section{Stability of $\mathrm{ZrS}_{3}$ flakes under different treatments}

Figures S2(a)-(e) show optical images of different $\mathrm{ZrS}_{3}$ flakes before (upper panels) and after (lower panels) heating in air to temperatures of $100{ }^{\circ} \mathrm{C}, 200{ }^{\circ} \mathrm{C}, 300{ }^{\circ} \mathrm{C}, 400{ }^{\circ} \mathrm{C}$ and $500{ }^{\circ} \mathrm{C}$, respectively. The heating time was kept constant at 10 minutes. The flakes do not show significant optical contrast change after $100{ }^{\circ} \mathrm{C}$ and $200{ }^{\circ} \mathrm{C}$ heat treatments, while a slight contrast change emerges at $300{ }^{\circ} \mathrm{C}$, indicating the onset of the oxidation process. The flake colour changes significantly at higher heating temperatures of $400{ }^{\circ} \mathrm{C}$ and $500{ }^{\circ} \mathrm{C}$, corresponding to a much higher oxidation rate.

Figure S3 shows optical images of $\mathrm{ZrS}_{3}$ flakes which were heated in $\mathrm{Ar} / \mathrm{H}_{2}$ mixed gas (19:1) flow at $400{ }^{\circ} \mathrm{C}$ for one hour. The flakes do not exhibit a significant contrast change. Therefore we conclude oxygen plays an important role in the contrast change of heated $\mathrm{ZrS}_{3}$ flakes.

To investigate the atmospheric stability of $\mathrm{ZrS}_{3}$ flakes at room temperature, we placed a chip that contained flakes in ambient conditions $\left(\sim 23{ }^{\circ} \mathrm{C}\right.$, relative humidity $\left.\sim 20 \%\right)$ for one month. Figure S4 show the optical micrographs and Raman spectra (in $E \perp b$ configuration) collected using the same parameters. Both data do not show a significant difference from the untreated samples, indicating that the oxidation process is quite slow at room temperature and hightemperature heating is crucial for the rapid oxidation of $\mathrm{ZrS}_{3}$ flakes. 


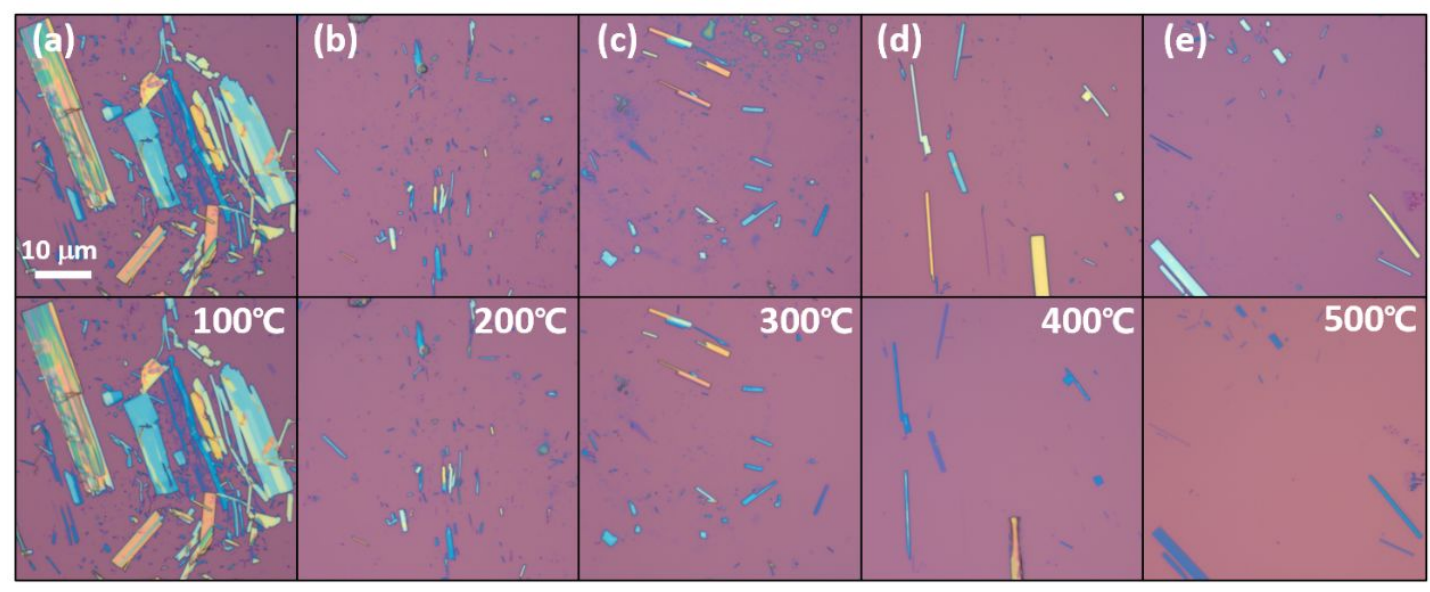

Figure S2. Optical images of $\mathrm{ZrS}_{3}$ flakes under different heating temperatures, exposed for 10 minutes. The scale bar in (a) applies to all images.

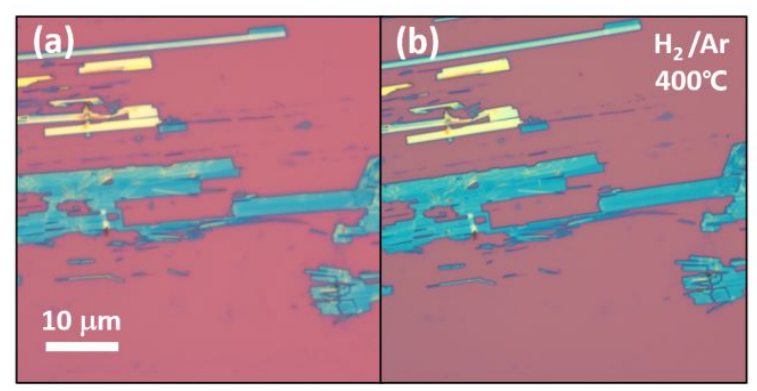

Figure S3. Optical images of $\mathrm{ZrS}_{3}$ flakes (a) before and (b) after $\mathrm{H}_{2} / \mathrm{Ar}$ gas annealing at $400{ }^{\circ} \mathrm{C}$ for one hour. The scale bar applies to both images.
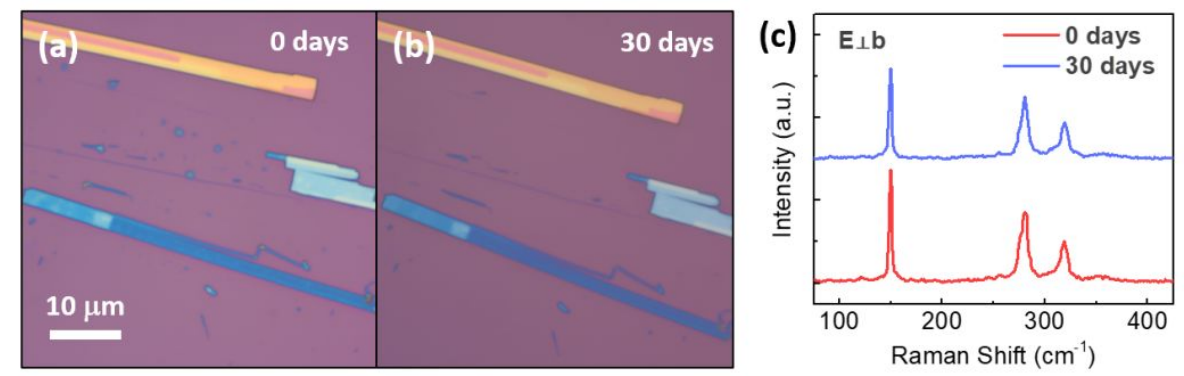

Figure S4. (a) and (b) are optical images of $\mathrm{ZrS}_{3}$ flakes captured before and after the samples were kept in ambient conditions for 30 days. The scale bar applies to both images. (c) Raman spectra of the same flake collected before and after the samples were kept in ambient condition for 30 days. No significant changes were observed in the peak amplitudes, positions or widths, suggesting the effect of surface oxidation was minimal.

\section{Stability of $\mathrm{ZrS}_{3}$ flakes at different substrates}


Figure S5 shows the reflective optical images of few-layered $\mathrm{ZrS}_{3}$ flakes transferred onto a transparent quartz substrate. In similarity to the case of standard $\mathrm{SiO}_{2}$ substrates, these flakes also lose their optical absorbance in the visible band after heating treatments, which corresponds to the proposed thermal oxidation process.

Figure S6 shows the PL spectra of $\mathrm{ZrS}_{3}$ flakes on various substrates before and after heating treatments. The transferred substrates include a $\mathrm{SiO}_{2}, \mathrm{Si}$ and sapphire. We also prepared a suspended flake. Although these measured flakes have different thicknesses, they all exhibit significant PL enhancement after heating (approximately 3-5 times stronger). Therefore, we conclude that the observed PL enhancement effect in thermally oxidised $\mathrm{ZrS}_{3}$ is independent of the underlying substrate. We also noticed that the PL peak positions exhibits slight shifts on different substrates, which might be attributed to strain effects.
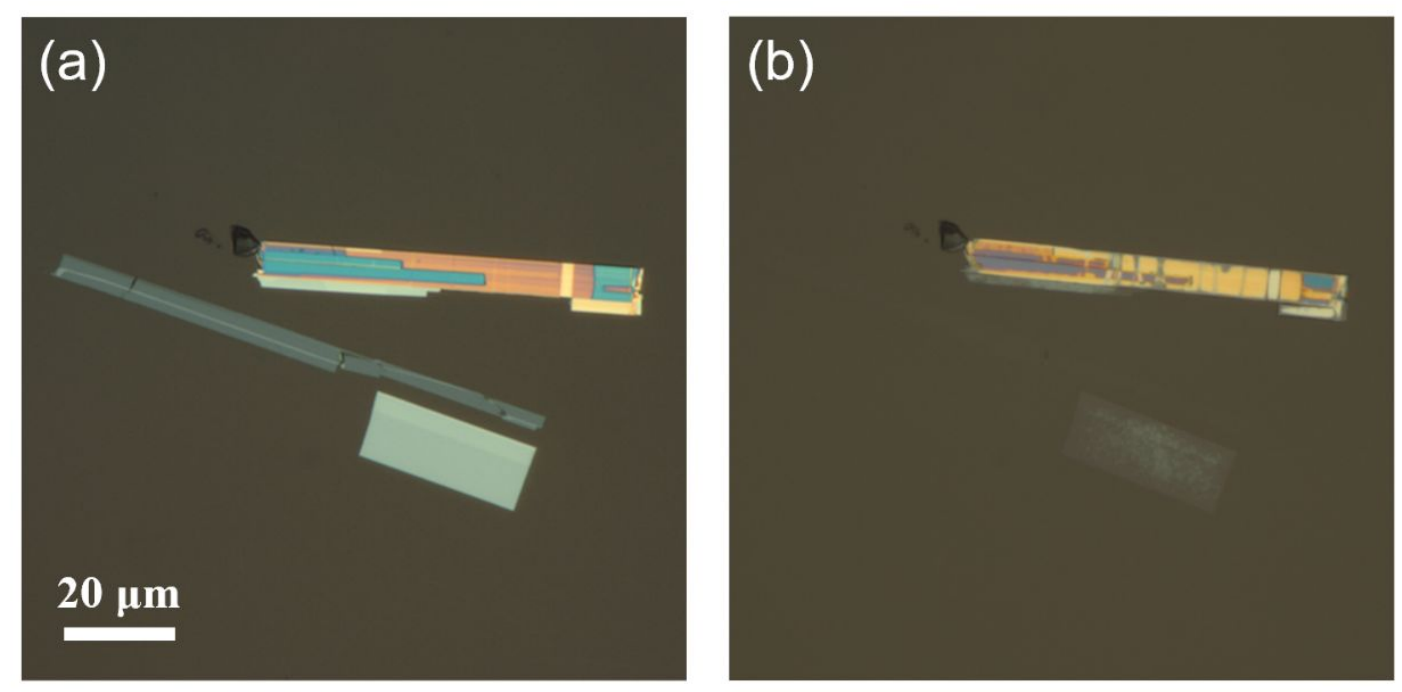

Figure S5. Reflective optical images of $\mathrm{ZrS}_{3}$ flakes transferred onto transparent quartz substrates (a) before and (b) after heating treatments $\left(350^{\circ} \mathrm{C}\right.$ for 10 minutes). The thin flakes look more "transparent" after heating treatment, indicating they are oxidised. 

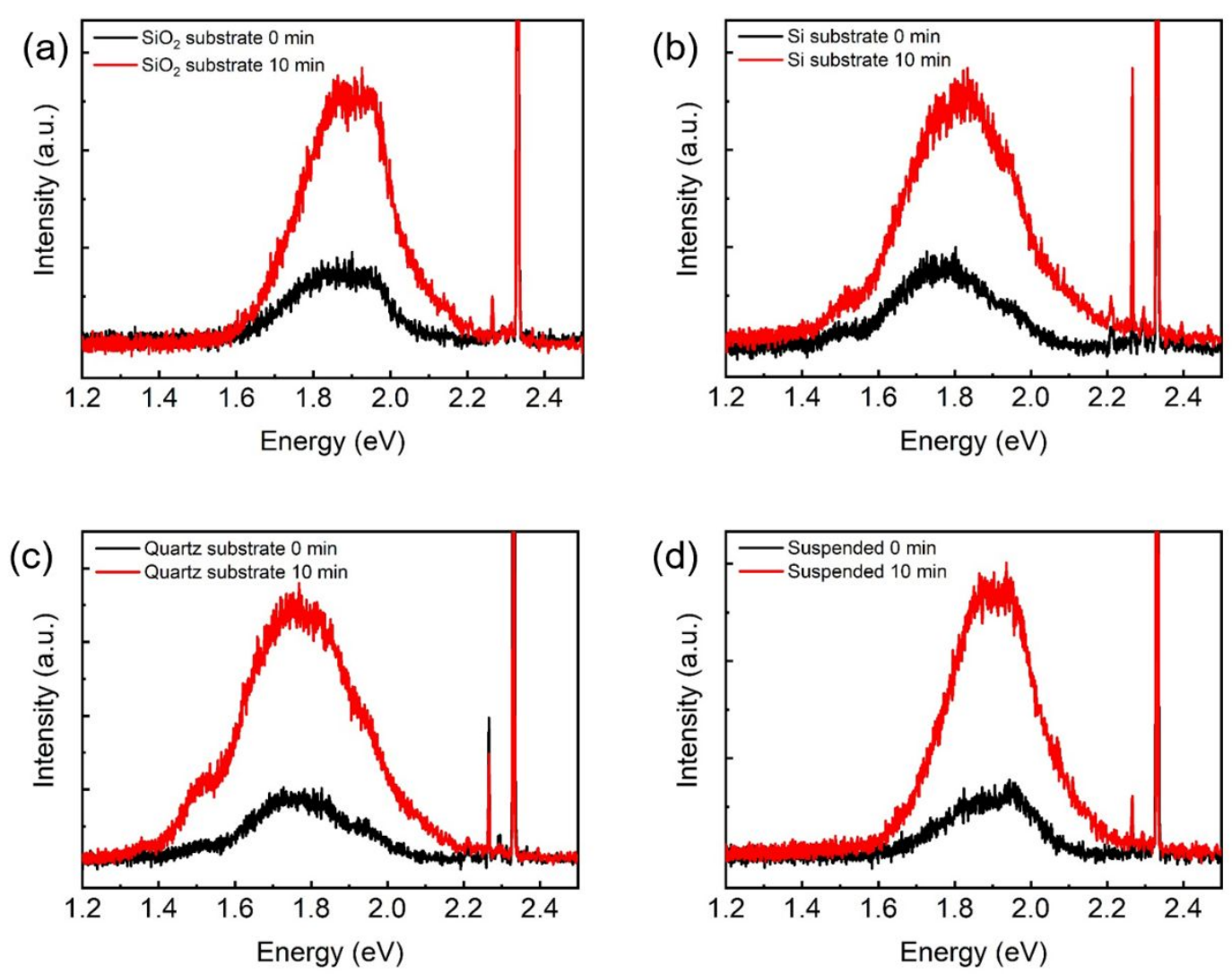

Figure S6. PL spectra of $\mathrm{ZrS}_{3}$ flakes before and after heating treatments. The flakes are transferred onto different substrates: (a) Si substrate with $285 \mathrm{~nm} \mathrm{SiO}$ layer. (b) Si substrate. (c) Quartz substrate. (d) Suspended flake with no supporting substrate.

\section{Calculation of the optical reflectivity of $\mathrm{ZrS}_{3}$ flakes}

We used a simple model, developed by Blake et. al for graphene layers on $\mathrm{SiO}_{2} / \mathrm{Si}_{\text {substrates }}{ }^{1}$, to calculate the expected optical contrast of $\mathrm{ZrS}_{3}$. As shown in Figure $\mathrm{S} 7$, a $\mathrm{ZrS}_{3}$ flake with thickness $d_{1}$ is placed on top of a Si substrate with an oxidised surface layer of thickness $d_{2}$. We only consider the case of normal light incidence from air onto this trilayer structure. The refractive index of air is $n_{0}=1$. For the trilayer structure, the values of their refractive indices are wavelength-dependent. For simplification, we choose a fixed value $(\lambda=550 \mathrm{~nm})$ for the calculation, which does not show significant variation from our experimental measurements. The values for common materials can be obtained from the online refractive index database (https://refractiveindex.info/). For the $\mathrm{Si}$ substrate and $\mathrm{SiO}_{2}$ layer, these are: $n_{3}(\lambda=550 \mathrm{~nm})$ $\approx 4.09-0.04 i$ and $n_{2}(\lambda=550 \mathrm{~nm}) \approx 1.46$, respectively. The refractive index of asexfoliated $\mathrm{ZrS}_{3}$ is crystal orientation-dependent, and for the $E \| b$ configuration, it has been reported to be: $n_{1}(\lambda=550 \mathrm{~nm}) \approx 3.4-0.1 i^{2,3}$. After the sulfide is completely converted to 
$\mathrm{ZrO}_{2}$, the refractive index changes to $n_{1}^{\prime}(\lambda=550 \mathrm{~nm}) \approx 2.17$. Therefore, the reflected light intensity can be written as:

$$
\begin{aligned}
I\left(n_{1}\right) & =\mid\left(r_{1} e^{i\left(\varphi_{1}+\varphi_{2}\right)}+r_{2} e^{-i\left(\varphi_{1}-\varphi_{2}\right)}+r_{3} e^{-i\left(\varphi_{1}+\varphi_{2}\right)}+r_{1} r_{2} r_{3} e^{i\left(\varphi_{1}-\varphi_{2}\right)}\right) \\
& \times\left.\left(e^{i\left(\varphi_{1}+\varphi_{2}\right)}+r_{1} r_{2} e^{-i\left(\varphi_{1}-\varphi_{2}\right)}+r_{1} r_{3} e^{-i\left(\varphi_{1}+\varphi_{2}\right)}+r_{2} r_{3} e^{i\left(\varphi_{1}-\varphi_{2}\right)}\right)^{-1}\right|^{2}
\end{aligned}
$$

where $r_{1}, r_{2}$ and $r_{3}$ are the relative indices of refraction, which are defined as:

$$
\begin{aligned}
& r_{1}=\frac{n_{0}-n_{1}}{n_{0}+n_{1}}, \\
& r_{2}=\frac{n_{1}-n_{2}}{n_{1}+n_{2}}, \\
& r_{3}=\frac{n_{2}-n_{3}}{n_{2}+n_{3}},
\end{aligned}
$$

$\varphi_{1}$ and $\varphi_{2}$ are the phase shift due to the optical path within intermedia, $\varphi_{1}=2 \pi \mathrm{n}_{1} \mathrm{~d}_{1} / \lambda$ and $\varphi_{2}$ $=2 \pi \mathrm{n}_{2} \mathrm{~d}_{2} / \lambda$. If we replace $n_{1}$ with $n_{0}$, we can obtain the reflected intensity $I\left(n_{0}\right)$ from the substrate. Therefore the optical contrast is defined as:

$$
C=\left(1-\frac{I\left(n_{1}\right)}{I\left(n_{0}\right)}\right) \times 100 \%
$$

air: $n_{0}=1$

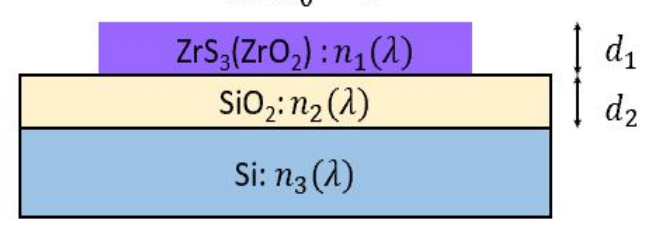

Figure S7. Sketch of the $\mathrm{ZrS}_{3} / \mathrm{SiO}_{2} / \mathrm{Si}$ stack used for the optical contrast calculation.

\section{Calculation of the stable phase of $\mathrm{ZrO}_{2}$ on the $\mathrm{ZrS}_{3}$ surface}

Density functional theory calculations were performed to identify the most stable interface for various phases of $\mathrm{ZrO}_{2}$ on $\mathrm{ZrS}_{3}$. Monoclinic, tetragonal and orthorhombic structures are presented in Figure S8, which are the most stable phases observed experimentally, among which the monoclinic phase has been identified to have the lowest bulk formation energy. The interface models were built matching the lattice constant of few-layer $\mathrm{ZrO}_{2}$ to bulk $\mathrm{ZrS}$. All three models share the same number of atoms so that the energy can be compared directly. The coordinates of the atoms were fully relaxed to calculate the total energy difference of various phases. The monoclinic interface has the lowest energy due to its good lattice mismatch and 
continuous symmetry across the interface. The energy differences are summarised in Table 1 in the main text. In contrast, large distortions are observed in the tetragonal and orthorhombic phases, making them less energetically favourable to form.

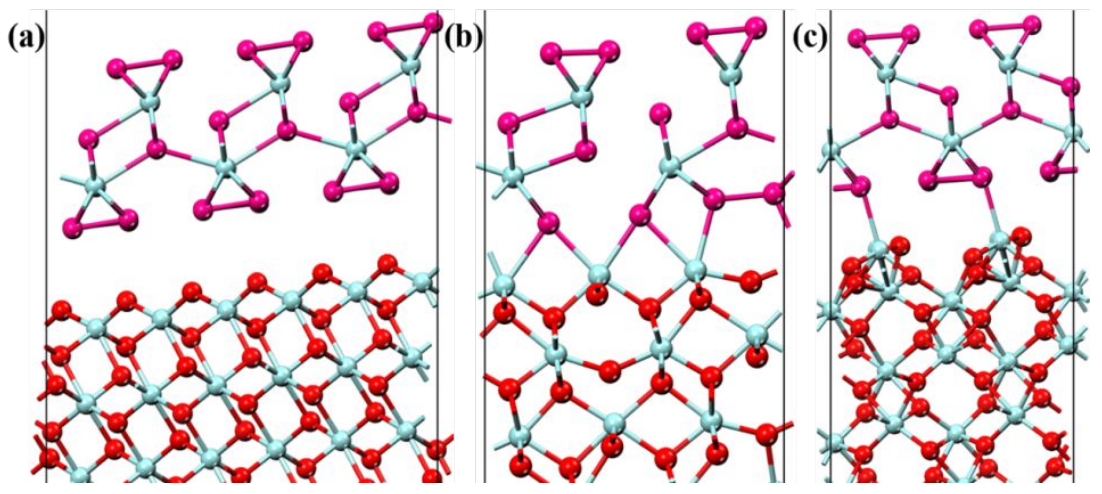

Figure S8. The atomic interface models used to determine the most stable phase of $\mathrm{ZrO}_{2}$ on top of bulk $\mathrm{ZrS}_{3}$. $\mathrm{Zr}$-cyan, S-red, O-pink. (a) The monoclinic phase of $\mathrm{ZrO}_{2}$. (b) The tetragonal phase of $\mathrm{ZrO}_{2}$. (c) The orthorhombic phase of $\mathrm{ZrO}_{2}$.

\section{Raman spectra of thermally-oxidized $\mathrm{ZrS}_{3}$}

Figure S9(a) shows the evolution of Raman spectra with heating for the same $10 \mathrm{~nm}$-thick $\mathrm{ZrS}_{3}$ flake, collected in the $E \| b$ configuration. The trend in time is similar to that of the spectra collected in the $E \perp b$ configuration (Figure 4(a) of the main text), i.e. the intensities of characteristic Raman peaks are reduced with increasing heating time and disappear totally after six minutes of heating. The additional peaks that correspond to the formation of $\mathrm{ZrO}_{2}$, which are observed in the $E \perp b$ configuration, are not seen here. This may be due to the intensity of $\mathrm{ZrS}_{3} \mathrm{~A}_{\mathrm{g}}$ modes being much strong in the $E \| b$ configuration than in $E \perp b$ configuration, obscuring the signal from the thin oxide peaks. Figures S9(b) and S9(c) show the evolution of Raman peak positions $\left(A_{g}^{5}\right.$ and $\left.A_{g}^{6}\right)$ with increasing heating times. The peak positions remain almost constant for the first five minutes and begin to exhibit small shifts $\left(<2 \mathrm{~cm}^{-1}\right)$ after six minutes of treatment. 

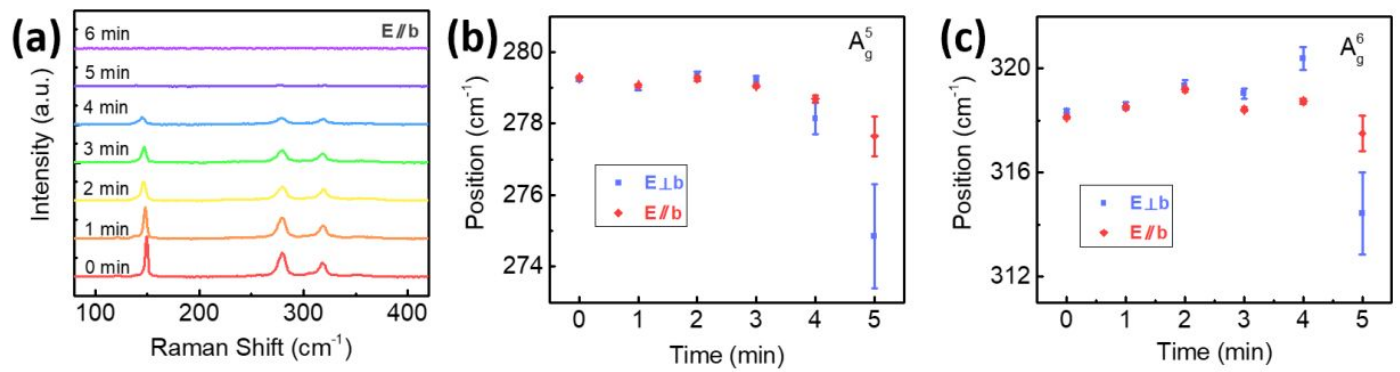

Figure S9. (a) Raman spectra of a $10 \mathrm{~nm}$-thick $\mathrm{ZrS}_{3}$ flake collected in the $E \| b$ configuration under different heating times. (b) The peak position of the $A_{g}^{5}$ mode as a function of heating time. (c) The peak position of the $A_{g}^{6}$ mode as a function of heating time.

\section{Angle-resolved PL emission from thermally oxidised $\mathrm{ZrS}_{3}$}

Figure S10 shows the photoluminescence spectra of a thermally oxidised $\mathrm{ZrS}_{3}$ flake collected under different laser polarization angles. The results show that, unlike pristine $\mathrm{ZrS}_{3}$ which exhibits anisotropic photoemission, the PL intensities from thermally oxidised $\mathrm{ZrS}_{3}$ collected across different laser polarization angles are almost the same, thus exhibiting isotropic photoemission.

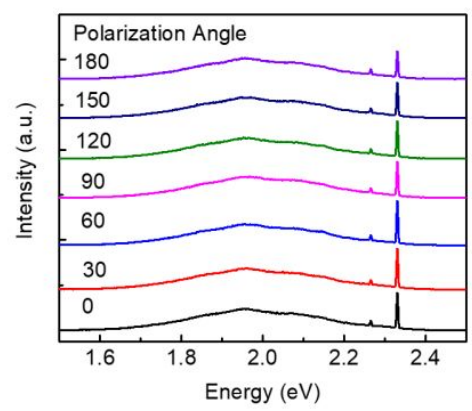

Figure S10. PL spectra of a thermally oxidised $\mathrm{ZrS}_{3}$ flake collected under different laser polarization angles from 0 to 180 degrees.

\section{Reference}

1. Blake, P.; Hill, E. W.; Neto, A. H. C.; Novoselov, K. S.; Jiang, D.; Yang, R.; Booth, T. J.; Geim, A. K., Applied Physics Letters 2007, 91 (6), 063124.

2. $\quad$ Kurita, S.; Tanaka, M.; Lévy, F., Phys. Rev. B 1993, 48 (3), 1356-1360.

3. Ahammed, R.; Rawat, A.; Jena, N.; Dimple; Mohanta, M. K.; De Sarkar, A., Applied Surface Science 2020, 499, 143894. 